\title{
Dokumentation
}

Axel Ermert*

\section{Zwischen Katalogisierung und Inhaltserschließung: Codierung von Dokumentarten - eine offene oder versteckte Aufgabe in Informationssystemen}

\author{
Nachbemerkungen zu einem nach wie vor aktuellen Thema
}

DOI 10.1515/iwp-2016-0051

Der vorstehend wiedergegebene Beitrag von Ingetraut Dahlberg mag den einen oder die andere womöglich zunächst etwas überraschen - vielleicht wegen des „Alters“ der behandelten Studie, weil sie „überholt“ sei oder aber, weil die Thematik sehr speziell oder gar „kleinkariert“ wirkt? Aber das ist sie nicht. Das Grundanliegen der Dokumentation ist seit ihrer Begründung vor 120 Jahren unverändert: Information aufzufinden (ggf. möglichst alle Information) zu einem vorgegebenen Thema oder einer speziellen Frage - unabhängig davon, wo sich diese Information befindet und in welcher äußeren Gestalt sie „verpackt“ ist. Obwohl um 1900 zunächst schwerpunktmäßig nur gedruckte, schriftliche Dokumente (das „Bibliotheksgut“ sowie Patentschriften) den Schwerpunkt der „Dokumentation“ bildeten, waren von Anfang an auch Mikrofilme, Museumsobjekte und all die anderen Dokumente gemeint.

Die sich groß entwickelnde, wissenschaftlich ausgerichtete Universalklassifikation „UDC / DK Universal Decimal Classification / Dezimalklassifikation“ - die Ingetraut Dahlberg als Beispiel erörtert - trug dem auch dadurch Rechnung, dass sie eine eigene „Hilfs“-Tafel „der Form“, also der Kennzeichnung der Dokumentarten, einführte.

*Kontaktperson: Axel Ermert, DGI-AKTS Arbeitskreis Terminologie und Sprachfragen, Institut für Museumsforschung, In der Halde 1, 14195 Berlin, E-Mail: a.ermert@smb.spk-berlin.de

\section{Welcher formale bibliographische Status, d.h. welchen Zugangs-/ Suchweg wählen - wo suchen?}

Information ist stets in mindestens einer „Box“ enthalten, denn jede nicht-flüchtige (dann z. B. nur mündliche) Information liegt in Form eines Dokuments vor (DatenbankDatensatz, Zeitschriftenartikel, Pressemitteilung, ...). Diese „Box“, also eigentlich dieses Dokument, hat bestimmte formale und inhaltliche Eigenschaften, die es kennzeichnen. Und diese Eigenschaften - nicht einmal die wesentlichen unter ihnen - sind oft keineswegs im Dokument-Titel enthalten oder auch äußerlich auf dem Dokument deutlich sichtbar zu erkennen. Darüber hinaus steckt in dieser ersten „Box“ u. U. oft noch eine weitere „Box“, nochmals eine eigens abgegrenzte, gekennzeichnete Passage innerhalb eines längeren Textes oder Bildes. Die „Volltextsuche“ der Suchmaschinen knüpft direkt an die digitale Textcharakteristik an und liefert entsprechend gleich die indexierten Treffer - sie ,durchschlägt‘ also, soweit von außen für den menschlichen Nutzer sichtbar, u. U. mehrere „Boxen“-Ebenen in einem Durchgang und gelangt gleich bis ans endgültige Ende, die gesuchte Information. Nach wie vor ist aber als häufiger Fall anzunehmen, dass die gewünschte Information für den menschlichen Nutzer noch nicht gleich in der ersten „Verpackung“/Box sichtbar ist, sondern erst in einer weiteren, in dieser Box enthaltenen (Unter-)Box, einer „Box in der Box“ - der berühmten russischen Puppe gleich -: also in dem Gesamttext (z. B. Buch), innerhalb dessen in einem Kapitel, innerhalb dessen in einem Absatz, usw. Stärker noch: in einer Anzeige, die in einem Zeitschriftenheft steht, das in einem Jahrgangsband gebunden ist, der innerhalb des kompletten Mikrofilms der mikroverfilmten Zeitschrift steht. Wenn eine dokumen- 
tarische Erschließung stattfinden soll - entweder schon vorab am Dokument (und dessen Strukturauszeichnung) oder mühsam im nachhinein durch die Erschließenden dann sollten diese „Boxen“ und „Unter-Boxen“ für die dokumentarische Erschließung (heute oft automatisiert) ansprechbar definiert sein. Schon wenn man nicht durch die erwähnte „Volltextsuche“, sondern - sei es auch immer noch digital - über bloße Nachweisfunktionen, über Verzeichnisse (z. B. digital aufrufbare Titellisten oder Inhaltsverzeichnisse) recherchiert, ist eine Kennzeichnung nützlich und sinnvoll, die angibt, welcher Art formal (damit auch: wo befindlich) und inhaltlich die Untereinheiten sind, eine Kennzeichnung also, die einen Hinweis auf spezielle Teileinheiten („Unter-Boxen“) geben, die die gesuchte Information enthalten könnten oder in denen sie sich dann wirklich vorfindet. Für unsere heute verfügbare Information, die in bestimmten Bereichen in einer nach wie vor überwältigend großen Menge von konventionellen, analogen Dokumenten („Boxen“, Datenträgern) gespeichert ist, gilt dies in besonderem Maße, denn diese Informationen oder Dokumente müssen physisch analog aufgesucht werden. Unabhängig davon, ob sie konventionell analog vorliegen oder unverändert in dieser gegebenen Form digitalisiert sind - der Weg zu den Boxen und Unterboxen muss auch physisch durchmessen werden: den Zeitschriftenband finden, das Heft aufschlagen, den „Kasten“ mit der Information ansteuern oder die nötige Seite aufschlagen ...

Die „Box“, in der die gesuchte Information vorliegt oder vermutet wird, kann nun sehr unterschiedlich beschaffen sein. Sie wird je nach ihrer Art aus bibliothekarischer/dokumentverzeichnender Sicht unterschiedlich „bibliographisch (z.B. selbstständig)“ eingeteilt und behandelt. Die Information kann in einem Lehrbuch, einem Zeitungsartikel, einem Loseblattwerk, einem Zeitschriftenbeitrag, einer Monographie, einem Flugblatt, einem Lexikon usw. „eingepackt“ sein ${ }^{1}$, in einem BlogEintrag, einer online zugänglichen Datenbank, auf einer Webseite, als Twitter-Nachricht, als einfaches PDF-Dokument, usw. In traditionellen (Bibliotheks-)Katalogen waren überhaupt nur bestimmte dieser Verpackungen (also: Dokumentarten) und manche nur durch den ihnen übergeordneten Titel auffindbar; und für diese „unselbständigen“ muss(te) man dann den Weg über die übergeordnete, tatsächliche „bibliographische Einheit“ gehen. Universitä-

1 Diese Dokumentarten werden bereits als Beispiele ausführlicher erörtert in Ermert, Axel; Port, Peter: Probleme bei der Erstellung und Übersetzung von Normwörterbüchern - dargestellt am Beispiel ISO 5127. In: Deutscher Dokumentartag 1984. München: Saur, 1985. S. 308-338. re Dissertationsschriften wurden auf verschiedene Weise behandelt, mal im gesonderten Katalog geführt, jeweils stets aber mit einem Vermerk auf der Titelangabe versehen. Oder in Handschriftensammlungen ist z. B. die Angabe der Dokumentart als „Hss.“, „Ms“ oder „ms“ (,Manuskript") oft schon als Teil der Signatur enthalten und so sofort ersichtlich. Der Bedarf nach genauerer (zunächst formaler) Kennzeichnung des Dokumenttyps und bestimmter Dokumenteigenschaften bestand jedenfalls in gewissem Maße bereits während der gesamten Zeitspanne, in der die Bibliotheken noch allein den weitaus größten Anteil an der Informationsversorgung innehatten; er ist heute noch ungleich größer. Im klassischen „Bibliotheksbereich" kam entscheidend der Übergang vom klassischen Bibliotheksmaterial hin auch zu „Sondermaterialien“ oder „Sondergebieten“ hinzu, die in allen großen wissenschaftlichen Bibliotheken anzutreffen waren und sind. Und die Dokumentartkennung (z.B. „Mikrofilm“, „Rara - Benutzung nur im Sonderlesesaal“, „Systemvoraussetzungen: PC ...") ist ein wichtiger Hinweis auch für die Benutzung, auf die u. U. besonderen Benutzungsanforderungen eines bestimmten Dokuments.

\section{Welche Art von Material - „Sondermaterial“?}

Die heutige Staatsbibliothek Preußischer Kulturbesitz in Berlin - wie etliche andere ebenfalls - begann nach 1914 auftragsgemäß, auch eine umfangreiche Sammlung von Kriegsschriften, Flugblättern, Propagandabroschüren anzulegen - ganz neue Dokumentgattungen ohne Vorläufer im damaligen System; und diese waren nur durch die angegebene übergeordnete Bezeichnung der "Sammlung“ überhaupt ersichtlich. Neue Dokumentgattungen im Bestand erzeugen neuartigen Bedarf an Dokumentartkennzeichnungen. Für die Arbeitenden in den Informationseinrichtungen kommt als ein Nebenaspekt noch hinzu, dass sie „Dokumente“ (Objektgruppen) verwaltungsmäßig unterschiedlich erfassen und behandeln und beispielsweise auch unterschiedlichen Erhaltungsstrategien unterwerfen müssen - eine formale Information, die zumeist, wenn vorhanden, in eigenen Verwaltungskatalogen und Datenbanken abgelegt war und ist ${ }^{2}$. Diese Unterscheidung wurde meist nicht als Teil der Dokumentbeschreibungen („Katalogisate“, Katalogeinträge) selbst aufgenommen (außer in

2 Die UDC-Anhängezahlen der Form/Dokumentart waren allerdings dafür wohl nicht ursprünglich gedacht. 
Museen), sondern war - allerdings nur vor Ort - lediglich dadurch sichtbar, dass unterschiedliche Dokumentarten schlicht in unterschiedlichen Benutzerkatalogen oder nur in Dienstkatalogen geführt wurden: ein Katalog „Monographien“, ein Katalog „Zeitschriften/Serien“, ggf. ein Katalog „Zeitschriftenaufsätze (unselbständige Publikationen)“, ein Katalog „Sondermaterialien“ oder „Tonträger“ oder „Mikrofilme“, Kennzeichnung des besonderen Sprachbereichs der Dokumente durch einen gesonderten Katalog wie „Russische Literatur/Slavica/Kyrillika“ usw. usf. Dies ist für die klassische analoge Suche (im Katalog) „,vor Ort“ (und für manchen Verwaltungsvorgang) vielleicht auch eine einigermaßen einfache und praktikable Lösung.

Auf die geschilderte Weise war und ist in der Tat die beim Vorgehen der Recherche zu treffende Auswahl in der Informationssuche/-beschaffung jedenfalls in gewisser Weise bereits stark von einer Vorsteuerung abhängig. Und überall da, wo eine Dokumentartkennzeichnung in gar keiner Weise anzutreffen war, konnten die Benutzer eine solche vorab bei der Auswahl helfende Information dann eben nur äußerlich, oder im „Bibliothekars-, Archivarsoder Forscherwissen “ in Erfahrung bringen. Bei den dann tatsächlich auf Anhieb auffindbaren bibliographischen „Verpackungen“ waren zwar immer schon einige formale Dokumentartmerkmale regelmäßig angegeben: um die wievielte Auflage (was Änderungen oder Popularität anzeigen kann), einen unveränderten Nachdruck, eine Lizenzausgabe, eine Übersetzung es sich handelt. Aber was hilft das, was hilft es bei der Suche nach bestimmten, mehr inhaltlich gefassten Dokumentarten, wenn es also darum geht, schon im vorhinein weitere Information zu erhalten über das, was einen an Informationsgestalt erwartet?

Und ein erheblicher Teil von Dokumenten konnte selbst auf keinem der bis hierher genannten Wege gefunden werden, weil er in diesen Katalogen überhaupt nicht verzeichnet wurde oder eine im Katalog nicht erkennbare Untereinheit bildete: Patente musste man grundsätzlich an anderer Stelle suchen, öffentlich ausgehängte (Werbe-, Wahl-)Plakate hätte man nirgends (nur versteckt vielleicht in Museen) verzeichnet gefunden.

\section{In welcher Weise wird die Information präsentiert - kondensiert, polemisch, gutachterlich, anweisungsorien- tiert, diskursiv, unmittelbar forschungsorientiert?}

Ein nächster Schritt, eine „formal-inhaltliche“ Angabe des Dokumenttyps und damit gewisser Dokumenteigenschaften, ist für viele Suchfragen und für bestimmte Forschungsinteressen essentiell wichtig und ausgesprochen nützlich: etwa für jemand, der ausschließlich Patente finden will oder vielleicht nur Globusse. Und die Feststellung und Einschätzung, um welche Art von Dokumenten es sich genau handelt, hat auch für die wissenschaftliche quellenkundliche Arbeit hohe Bedeutung zur Einordnung, der Einschätzung der Verlässlichkeit und Quellenbedeutung des Dokuments. Eine vorab verfügbare Dokumentartkennung ersetzt dieses Studium nicht, gibt aber eine zusätzliche Spezifizierung, ob ein bestimmter Informationsträger, ein bestimmtes Dokument für die beabsichtigte Arbeit die richtige Wahl sein mag.

Und es spielt im weiteren nun in der Tat die genauere Art, z.B. das Genre, des Dokuments eine wichtige Rolle: dass ein Dokument, das als „Wörterbuch“ oder "Glossar“ ausgewiesen ist, Information auf seine dadurch bezeichnete Art bereitstellen würde, war vielleicht durch den Titel noch ausgewiesen und von den Suchenden erkennbar und erwartbar. Manche Dokumente enthalten einen Hinweis auf ihre Dokumentart in ihrer Titelei, aber viele andere auch nicht. Ein ausschließlicher Sachtitel, wie er bei einer sehr großen Zahl von Dokumenten vorkommt, erlaubt aber jedenfalls zunächst gar keinen näheren Aufschluss, welche Art der Informationsdarbietung (Dokumentart) einen hier erwartet. Z. B. erst der häufig schon eigens dem Dokumenttitel noch hinzugefügte Zusatz „Roman“ zeigte für bestimmte Dokumente nach außen klar an, worum es sich handelt. Und bei einer bloßen Titelangabe wie „Die Venus von Milo" ist nun in der Tat in gar keiner Weise mehr von vornherein klar, ob es sich um ein (archäologisches/kunsthistorisches) Sachbuch, ein Gemälde, eine Skulptur, einen Forschungsaufsatz aus einer kunsthistorischen Zeitschrift, einen Leserbrief oder vielleicht gar um einen restaurierungstechnischen Forschungsreport zu einer erfolgten konservatorischen Untersuchung handelt. Einem solchen Fehlen von Information entgegen wirkend wurde seit den 1920er Jahren in der Titelangabe in Zeitschriften mit technisch-wissenschaftlichen Aufsätzen und in vergleichbaren 
Publikationen die Dokumentartcodierung (der UDC) der inhaltscodierenden Notation mit angefügt - lange Zeit auch in den „Nachrichten für Dokumentation“ - , so dass schon auf diese Weise nach außen auch sichtbar ist, in welcher Dokumentart die Information präsentiert wird. Und ob eine Angabe wie „Hochschulschrift“ als Dokumentartangabe eine Doktordissertation oder ähnliche „Hochschulschrift“ dieser Art bezeichnet - und nicht etwa eine Schrift, die die Universität aus speziellen Feieranlässen als eigene Festschrift herausgibt - , ist für den Informationssuchenden wichtig zu wissen: die genauere Dokumentartangabe zeigt schon im Vorhinein präziser die Wahrscheinlichkeit an, ob der beabsichtigte Nutzen aus diesem Dokument zu ziehen sein mag. In diesem Beispiel der „Hochschulschrift“ kommt so auch noch die Notwendigkeit hinzu, die Dokumentart nicht nur anzugeben, sondern auch durch eine Erläuterung zu definieren (siehe unten Seite 203). In jedem Fall - nehmen wir auch noch einmal das Web als Beispiel: Die Entscheidung, ob ich einen bestimmten Treffer im Web anklicken soll, fällt manchmal doch leichter und schneller, wenn ich schon in der Vorschauanzeige einen Hinweis erhalte („Pariser Weltausstellung 1900.“ Aha. Also ein Ausstellungskatalog? Oh, nein: „Flugblatt“).

Ein solcher Bedarf aber meldet sich, wie gesagt, in jedem Falle fest und unabweisbar in dem Augenblick, wo man bei der Recherche als Basis für Informationsversorgung und -nachfrage die wesentlich breitere Grundlage „alle Arten von Dokumenten“ wählt - und ggf. gleichzeitig ein Universalverzeichnis (wie z.B. eine „Bibliographie“ oder „Europeana“) als Nachweisinstrument unabhängig von der Bindung an Standort(angab)en nutzt. Denn für „remote information retrieval“ (dessen erster Schritt ja schon die Benutzung eines jeweiligen Katalogs war und ist) hilft die oben erwähnte klassische Unterbringung in und Konsultierung von unterschiedlichen Katalogen nur bedingt. Gewünscht vom Katalog in der webbasierten Recherche ist ja, heute ein möglichst breites Verzeichnis („one-stop shop“) zu bieten und in diesem unabhängig und weit entfernt von den physischen Standorten der Dokumente eine „Ferndiagnose“ durchführen zu können, ob in dem angegebenen Dokument die gesuchte Information vorhanden sein mag. Und da ist u.a. auch die Suche in einem möglichst größeren gemeinsamen Pool von Vorteil, weil sonst aufwändig ermittelt werden muss, in wie vielen anderen Katalogen man noch der gerade gegebenen eigenen Fragestellung nachgehen muss. Für eine solche „Suche von Ferne" und in einem möglichst großen Angebotspool ist aber eine genaue Kennzeichnung der jeweils erzielten „Treffer“ auch nach Dokumentart-Eigenschaft ein außerordentlicher „Mehrwert“, der Umwege und Re- trievalballast vermeidet. Genau dies fügt eine „Dokumentartkennzeichnung“ der Dokumentverzeichnung in einem (Bibliotheks-) Katalog, einer Bibliographie usw. hinzu, so dass im Instrument der UDC, aber ebenso in anderen Klassifikationen, auch ein mehr oder weniger vollständiger Bereich der Klassifizierung der Dokumentart bereitstehen sollte. Dewey DDC (bereits ab Beginn, 1876), die UDC und auch andere große Klassifikationssysteme seither (wie Bliss Bibl. Classif., BBK Bibliothekarisch-bibliographische Klassifikation) führen solche besonderen (Hilfs-)Tabellen entweder am Anfang oder am Ende, u.a. für die Dokumentartangabe. Eine Ausnahme bildet wohl vor allem Ranganathans „Colon Classification“, wo die Dokumentartangaben bei den einzelnen Sachgebieten mit eingebaut sind (z. B. (1P1)).

Eine solches Instrumentarium für Dokumentartkennzeichnung bereit zu stellen, ist inzwischen in unterschiedlichem Ausmaß in verschiedenen Klassifikationssystemen, Thesauri, und Online-Portalen geschehen. Mittlerweile geben modernere Kataloge („Discovery Systeme“) jeweils eine Grundinformation zur Art der aufgewiesenen ,Ressource“ an: wurde man Anfang der 1990er Jahre noch erstaunt angeschaut „Ja wollen Sie denn in unserem (Bibliotheks-) Katalog jedem Eintrag die Kennung „Buch“ hinzusetzen?“, findet man heute dort viele Dokumente bereits durch ein Icon oder ein Wort gekennzeichnet: „elektronische Ressource“, „Hörbuch“, „Textdokument“, „Spiel“, „Tonträger“, „Mikrofiche-Sammlung“ usw. In Online-Portalen wird häufig auch eine „Facettierung“ der Suche angeboten, d.h. man kann nach Zeitraum, Content Provider, geographischer Region und ähnlichen formalen Merkmalen filtern, suchen, auswählen. Für die unterschiedlichen Dokumenteigenschaften, die die „Dokumentart“ ausmachen, ist dies z.Zt. noch weniger der Fall als für andere Merkmale; immerhin stellen etliche Online-Portale (wie „Deutsche Digitale Bibliothek“ oder „Europeana“) - insbesondere solche, die „Mischinformation“, also unterschiedliche Dokumentarten, nebeneinander und gemeinsam bereitstellen - Anfangskategorien wie „textual resource“, „audio resource“, ,image“ usw. bereit. Für tiefer gehende Hinweise zur Dokumentart - im oben skizzierten Sinne - fehlt oft schlicht die Information in den angelieferten Metadaten, also den zugrunde liegenden Erschließungsdaten. In einigen Thesauri wie dem Thesaurus Sozialwissenschaften, dem Standardthesaurus Wirtschaft oder dem INFODATA Thesaurus finden wir sehr wenige, sehr karg begrenzte Einträge, die die Möglichkeit zur Indexierung der Dokumentart der jeweils vorliegenden Informationsquelle bereitstellen. Eine systematische Gliederung nach den Aspekten von „Dokumentart“ finden wir in kaum einem auf Anhieb gewärtigen Lexikon der Bibliotheks- oder 
Informationswissenschaft - allerdings in ,alten“ DDR-Ausbildungsheften zur „Informationsquellenlehre“.”3

\section{Wie vorgehen - was angeben?}

Auf die durch die Dahlberg-Studie ${ }^{4}$ skizzierte Situation, die nachhaltig hervorgehobene Notwendigkeit einer Systematisierung, genauen Auflistung und Kennzeichnung angesichts der großen Variationsbreite/Fülle von Dokumentarten - und auf die verständliche, in den UDC-Tafeln der „Allgemeinen Anhängezahlen“ deutlich sichtbare, auch durch das direkte Aufgreifen jeweils gerade aktueller CodeBedürfnisse hervorgerufene Selektivität und ein wenig auch Unausgewogenheit - war schon zuvor und wurde in der Folgezeit in verschiedenen Hinsichten reagiert. Die damit also hier ausführlich beschriebenen und entwickelten Dokumentartbezeichnungen treffen wir in der Tradition der letzten Jahrzehnte in der Bibliothekskatalogisierung als „Formschlagwörter“. Und das Sachkatalogisierungssystem „Eppelsheimer“ hatte bereits seit den späten 1920er Jahren reagiert, indem es den Katalog an der jeweiligen Sachstelle durch zusätzliche Reiter untergliedert, z. B. „Jahresberichte“, „Bibliographie“, „Monographie“, „Bildband“, „Aufsatz“, „Stand der Forschung: Überblick“, „Polemik zum Thema“ usw., viele Dokumente also ausdrücklich nach bestimmten Form-Inhalts-Merkmalen ausweist. $^{5}$ Das UNESCO-,Vocabularium bibliothecarii“ (1953/1962) als Auszug aller bibliothekarisch-dokumenta-

3 Freytag, Jürgen: Einführung in die Informationsquellen- und Informationsmittelkunde: Lehrbrief. - Berlin: Institut für Bibliothekswissenschaft und wissenschaftliche Information, Humboldt-Universität, 1974. 64 S. - Freytag, Jürgen: Informationsquellenkunde: Teil 1: Dokumentale und nichtdokumentale Informationsquellen und -mittel. Lehrbrief. 3. Aufl. Berlin: Institut für Bibliothekswissenschaft und wissenschaftliche Information, Humboldt-Universität, 1986. 112 S. Freytag, Jürgen: Informationsquellenlehre: Teil 1: Theoretische Grundlagen. Lehrbrief. 8., vollst. überarb. Auflage. Berlin: Institut für Bibliothekswissenschaft und wissenschaftliche Information, Humboldt-Universität, 1989. 61 S. - Ahnis, Gabriele: Informationsquellen und -mittel: Bibliographische, analytische und synthetische Informationsmittel. Berlin: Fachschule für wiss. Information und wiss. Bibliothekswesen, 1981. $85 \mathrm{~S}$.

4 Dahlberg, Ingetraut: Vorschlag für eine Klassifikation der Dokumentarten und der Dokumentenkunde $=$ Proposal for a classification of kinds of documents and documentology. Frankfurt/M.: Deutsche Gesellschaft für Dokumentation (DGD), 1970. 37 S.

5 Siehe hierzu: Riplinger, Thomas: Die Bedeutung der Methode Eppelsheimer für Theorie und Praxis der bibliothekarischen und dokumentarischen Sacherschließung. In: Bibliothek - Forschung und Praxis, 28 (2004)2, S. 253-262, besonders Abschnitt 5, S. 253 f. risch relevanten Teile aus der UDC listete noch einmal komplett alle Dokumentarten und -teile auf, aufsteigend nach ihrer UDC-Nummer, und war insofern ein wichtiges Hilfsmittel, aber keine Angebotserweiterung. Die „TID“ (1975) ${ }^{6}$ verschob das Kapitel „Dokumentarten“ auf ihre Folgeausgabe, die 1998 abgeschlossen war und 2006 erschien. Der Bedeutung der äußeren Form und inhaltlichen Darstellungsweise des Dokuments für seine Verzeichnung und Wiederauffindbarkeit nach den klassischen großen Bibliothekskatalogen trug Rechnung eine umfangreiche Studie und Arbeitsanleitung von Helmut Allischewski, „Retrieval nach Preußischen Instruktionen“. ${ }^{7}$ In ihr gibt der Autor nicht nur eine umfangreiche, detaillierte Studie von mehr als 400 verschiedenen Dokumentarten (formal/inhaltlich), wie sie vorkommen und in Bibliothekskatalogen verzeichnet werden können. Sondern er zeigt vor allem bei jeder einzelnen Dokumentart, welche Bedeutung ihr für die „Einordnungsentscheidung“, d.h. für die Katalogisierung und das entsprechende Wiederauffinden in den lange Zeit im deutschen Sprachraum vorherrschenden Katalogen nach „PI Preußischen Instruktionen für die alphabetische Katalogisierung“ (1899) zukommt, und wie vorzugehen ist. Codierungen bereit zu stellen ist jedoch nicht sein Thema. Im Gefolge der die PI ab 1976 ablösenden RAK „Regeln für die alphabetische Katalogisierung“ bzw. AACR „Anglo-American Cataloging Rules“" wurden auch digitale Kataloge und Normdateien aufgebaut, in denen für die Sachkatalogisierung der Werke nicht nur Norm-Schlagwörter, sondern innerhalb dieser auch „Formschlagwörter“ z. B. nach RSWK ${ }^{8}$ oder DIN 31631 Teil 2 zur Kennzeichnung bestimmter Dokumentarten/Dokumentformen eingerichtet wurden - die selbst auch mit meist zweistelligen alphabetischen und/ oder numerischen Kurzkennungen versehen sind. Ein Teil der Liste aus der Dahlberg-Studie ist von ihnen abgedeckt. Spezielle solcher Formschlagwörter finden sich, für

6 Terminologie der Information und Dokumentation [TID]/KTS der DGD, Red. Ulrich Neveling, Gernot Wersig. München: Saur, 1975. - 2. Ausgabe: DGI. Frankfurt am Main, 2006. 124 S.

7 Allischewski, Helmut: Retrieval nach Preußischen Instruktionen: Darstellung der Rechercheprobleme in ,preußisch“ geführten Katalogen anhand einer Systematik der Schriftenklassen. Wiesbaden: Reichert, 1982. XX, 288 S.

8 Siehe z.B. Regeln für den Schlagwortkatalog (RSWK). Anlage 6: Liste der Formschlagwörter. [Enthält ungefähr 155 Formschlagwörter]. http://d-nb.info/1022924354/34 [30.6.2016]. Ein Einstieg in die Formschlagwörter der GND Gemeinsame Normdatei ist auch möglich über: https://portal.dnb.de/opac.htm?method=showOptions bzw. über die ,Einfache Suche“ (mit beliebigem Sachbegriff, hier „Essen“): https://portal.dnb.de/opac.htm?query=essen\&method=simpleSearch 
die verfeinerte Erfassung von Beständen, zusätzlich im „TPRO Thesaurus der Provenienzbegriffe“. 9

Die Grundlage der Dokumentartcodierungen/Allgemeinen Anhängezahlen (siehe unten) findet sich bereits seit den Anfängen der UDC als System (ab etwa 1900), jedoch in - zumal aus heutiger Sicht - rudimentärer und aus heutiger Sicht - oft etwas ungleichmäßiger/ungleichgewichtiger Weise. Zunächst ganz an Deweys DDC orientiert und diese übernehmend, enthielt die UDC von Anfang an die Klasse „0 Wissenschaft und Kenntnisse im allgemeinen“, darin Abteilungen über „Schriftsysteme“, Bibliographien aller Art, Handschriften, Zeitungen (sowie das Bibliotheks- und Museumswesen im allgemeinen). Hier finden sich also bereits Notationen, mit denen einige, aber nur sehr ausgewählte „Dokumentarten“ gekennzeichnet werden können, zunächst die klassischen, „selbständigen" typischen Bibliotheksdokumente (exemplarisch: die „Bibliographie(n)“), in Bibliotheken als physisch selbständige Einheiten verzeichnete Dokumentarten. Hinzu kommen, manchmal am Ende aufgelistet (in der UDC-Ausgabe von 1905 sind es etwa zehn Einträge für Aspekte), später dieser Haupttafel „0“ dann vorgeschaltet, noch allgemeiner die „Anhängezahlen“ in der Gestalt „(0 ...)“., und damit eine viel umfangreichere Liste. Sie erfasst jetzt nicht mehr vor allem klassische „Bibliographien“, sondern z. B. (inhaltlich) „Bücher für Kinder, Rezepte, Formelsammlungen (als Polygraphien)“, oder (formal) „Broschüren, Sonderdrucke, Dissertationen, Aufsätze in Tageszeitungen, bibliographische Inhaltsangaben, Museumsstücke, Schallplatten, aufgeklebte Dokumente: Bilder - Marken“ usw. usw. Dies ist also ein deutlicher Schritt in Richtung auf Erfassen/Abdecken aller Dokumentarten und unabhängig von ihrer bibliographischen Form. Der „literarische, künstlerische, wissenschaftliche Schwindel“ (088.5) ist immerhin von Anfang an stets dabei - er war und ist wohl wichtig genug, um eigens aufgeführt zu werden. Und diese ausführlicher gewordene Liste der „Allgemeinen Anhängezahlen der Form“ ändert sich im Grundsatz nicht wesentlich, sie ist (kürzer) in gleicher Form enthalten etwa in der dreisprachigen Mittleren Ausgabe DK von 1958, und (ein wenig erweitert, z. B. um „Privatbriefe“, „Dienstschreiben“, „Abreißkalender“) in den deutschen Ausgaben

9 T-PRO Thesaurus der Provenienzbegriffe (65 Einträge) http://prove nienz.gbv.de/T-PRO_Thesaurus_der_Provenienzbegriffe. - Genre terms: A thesaurus for use in rare book and special collections cataloguing/Association of College and research Libraries (ACRL). - Chicago, 1991. - 78 p. - http://rbms.info/vocabularies/genre/alphabetical_ list.htm. Für eine spezielle Art kirchlicher, religiöser Dokumente: List of uniform titles for liturgical works of the Latin rites of the catholic church/IFLA Office for UBC. München: Saur, 1981. 17 S.
1967 - also kurz bevor die Dahlberg-Studie begonnen wurde -, 1973, 1978. Ein wesentlicher Schritt voran ist 1970 nun der strenger, durchweg systematisierende und nach inhaltlicher Beschaffenheit der Dokumente orientierte Ansatz in der Dahlberg-Studie, in dem sie in „036-038 Dokumente nach intendierter Funktion" (Dahlberg-Beitrag in diesem Heft, Seite 198-199) eine wesentlich umfangreichere, ausführlichere und gleichgewichtige, zum ersten Mal systematisch unter speziell dokumentarischen (= inhaltserschließenden), begriffssystematischen Kriterien aufgestellte Klassenbildung der für verschiedenste Anwendungsbereiche entstehenden, relevanten Dokumentarten gibt (und zahlreiche, in der Praxis wichtige ,kleine' Dokumentarten hinzufügt, besonders auch aus dem nicht streng bibliographischen Bereich). Als solche systematischen Klassen nennt sie etwa:

- „Dokumente mit Beweisfunktion“

- „Dokumente mit Ankündigungs- und Werbefunktion“

- „Dokumente mit Beratungs- und Vorschriftenfunktion"

- „Dokumente mit emotionellem Charakter“

- „Zum Ausfüllen bestimmte Dokumente“

- „Literarische Dokumente“

- in gleicher Weise hätte man (ansatzweise beginnt das schon in einigen der UDC-Ausgaben) z.B. auch den systematischen Aspekt des Schwierigkeits-/Verständlichkeitsgrades von Dokumenten hier mit aufnehmen können: „populärwissenschaftlich/...“ - wenn man eine einigermaßen klare definitorische Vorstellung im Kopf hat, was das ist

usw. (wie es die Norm ISO 5127 in ihrem kurzen Abschnitt „Documents by originator and intended purpose“ ebenfalls unternimmt - siehe unten). Zum gegenwärtigen Stand der UDC-Dokumentartcodierung/Anhängezahlen: In der UDC/DK verwirklicht finden wir heute (aktuellste, englische Ausgabe von $2005^{10}$ ) 6 Seiten „Tafel 1d Allgemeine Anhängezahlen der Form“, in der die Gliederung u.a. zeigt:

- (0.02) Documents according to physical form (large document, one-colour document, paperback editions, pirated editions, ... )

- (0.03) Documents according to method of production (handwritten, typescript, machine-readable/digital, photocopies, printed proofs, ... )

10 UDC Universal Decimal Classification: Standard Edition: Fully updated third edition. 2 Bände (Systematic tables; Alphabetical Index). London: British Standard Edition, 2005. 898, 526 S. 
- (0.04) Documents according to stage of production (draft, reprinted, abridged, ... )

- (0.05) Documents for particular kinds of users (children, women, ... )

- (0.06) Documents by level of exposition and availability

- (038) Dictionaries, (044) Correspondence, letters (046) Newspaper articles

- (05) Serial publications (07) Documents for instruction, teaching, study, training

- (083) Technical and normative documents (forms, tables, standards, inventories/catalogues, plans, directions for use, ... )

- (084) Images, pictorial, graphic documents (films, sound films, survey photographs, maps, .. )

- (085) Publications of business firms (trade catalogues, price lists, ... )

- (086) 3-dimensional, solid documents (specimens, reliefs, models, audio documents, videodiscs, .. )

- (088) Attestations; special-purpose documents (certificates - of property, licenses, passports, contracts, trademarks, patents, ... )

- (09) Legal and historical sources (Gesetze, Verordnungen, ... )

aber auch, beispielsweise, in den Haupttafeln (in einigen Fällen doppelt: als Anhängezahl und als Haupt-Notation): Riddles 389.6, Folk songs 389.8, Monuments 725.94, Flags 355.15 Military decorations, medals 355.134.2, Coins 671.41 (336.743) (737.1), Medals, plaques 671.42 (737.2), Types of literary work (books, newspapers, letters, archives) 347.781.5, Graphic arts: Drawings, lithographs, engravings, photographs 347.783, Products of printing 655.3.066, (printed) Books, pamphlets 655.3.066.11; advertisement matter 655.3.066.23, Posters 655.3.066.24 Calendars 655.3.066.26, Postcards 655.3.066.32 Tickets 655.3.066.34 Forms 655.3.066.35, Currencies, banknotes, securities 655.3.066.36 Maps, globes 655.3.066.43, Author's copy 655.527, Illustrations 655.533 Binding[s] 655.534, Advertising jingles 659.123; Documentary photographic records 77.03 (hier wäre eine erläuternde Definition hilfreich), Photographs of natural objects 77.031, Photographs of artificial, man-made objects 77.033, Daylight exposures 77.051, cartes-de-visite photographs 77.068.112, Libretti 78.089.1, Scores, parts 78.089.6; "heroes" (films) 791-51, ["biopic" films] 791.227, Theatre pieces, plays (solo, full-cast, .. ) 792.08, Poetry, poems, verse $82-1$, descriptions of travels 82-992, (archaeological) Stone and bone objects 903.01, Burial registers 929.53. Fernsehfilme würden durch Kombination wiedergegeben werden: 654.197 (Rundfunk, TV): 792.08 (Theaterstück) usw.
An dieser Stelle ist ein noch ausgreifenderes Weiterdenken möglich und sinnvoll. Da im Sinne Paul Otlets und der Dokumentationstheorie natürlich auch materielle Objekte in einer bestimmten Funktion (z.B. als Museumsobjekte oder Belegexemplare) die Qualität von „Dokument" haben, eröffnet sich eine große Bandbreite weiterer möglicher oder notwendiger Dokumentartkennzeichnungen. Auch wenn man sie auf die höheren Allgemeinbegriffe beschränkt, ergeben sich erhebliche Erweiterungen. Spezialklassifikationen etwa aus dem Museumsbereich geben hier eine erste Übersicht, etwa die „Nomenclature 3.0 for Museum Cataloging“11, die ganz nach Funktion genau im Sinne der Dahlberg'schen Studie Dokumentarttypen für dreidimensionale/korporale Dokumente angibt: 20 Hauptgruppen wie „Communication artefacts“, „Transportation artefacts" usw., und unterhalb dieser dann Feinuntergliederungen bis zu Individualbegriffen direkt für einen Objekttyp, etwa „Hammer“. Diese sind natürlich für eine Universalklassifikation wie die UDC zu ausführlich, geben aber eine interessante Richtung der Weiterentwicklung der Dokumentartkennzeichnungen an.

\section{Dokumentartcodierung - warum?}

Wie jede andere Information unterscheidet sich auch die in Katalogen und Verzeichnissen gebotene Information über Dokumente nach der grundlegenden kategorialen Unterschiedlichkeit, die allen Welt-Entitäten zukommt: üblicherweise den fünf (bzw. sieben) Grundkategorien: wer? was? wie? wo? wann? womit? warum - wofür? Diese Aspekte von Information geben u. a. Klassifikationen - in unserem Fall hier die UDC - wieder bzw. stellen das Hilfsmittel dazu bereit: das „Was“ ist Gegenstand der gesamten Klassifikation in ihren (Haupt-)Tafeln, die systematische Aufführung aller Sachinhalte und Wissensdisziplinen. Darin und dabei finden dann auch „Wann“ (Zeitangaben, geschichtliche Perioden) und „Wo“ (Auflistung der relevanten Örtlichkeiten) ihren Platz. Die „Allgemeinen Anhängezahlen“ zeigen dann eine der Formen des „Wie“. Sie sind dafür eingeführt, im Rahmen dieser grundsätzlichen Strukturiertheit einer Klassifikation an jede beliebige Inhaltskennzeichnung seitens der Klassifikation angehängt werden zu können - sie sind also nicht in eine bestimmte Stelle in der Hierarchie eingebaut, sondern können überall angefügt werden: weil sie Gegebenheiten ausdrücken, die

11 Nomenclature 3.0 for Museum Cataloging: Third edition of Robert Chenhall's system. Edited by Paul Boucier. American Association for Stae and Local History (AASLH). Lanham; New York: Alta Mira Press, 2010.716 S. 
im Zusammenhang mit jedem inhaltlichen Sachverhalt vorkommen (können). Die fünf oder sieben Grundkategorien sind ja gerade deshalb solche Grundkategorien, weil sie jedem Gegenstand, den es in der menschlichen Erfahrungswelt gibt, notwendig und immer (inhärent) zukommen. Und das „Wie“ - der Information, also ihr Vorliegen in welcher Art von Dokument -: das lässt sich so mittels der Anhängezahlen ebenfalls durch die Klassifikation systematisch kennzeichnen.

Im Rahmen dieser „Wie“-Angabe eröffnet sich dabei eine doppelte Möglichkeit, wie Ingetraut Dahlberg (Beitrag in diesem Heft der IWP, Seite 9) deutlich ins Blickfeld rückt: dass für die speziellen Notationen der Klassifikation, die „Allgemeinen Anhängezahlen (hier: der Form)“, stets „die zweifache Verwendungsmöglichkeit, nämlich einerseits, wenn Information über ein Dokument ... durch eine Notation codiert wird, und andererseits, wenn etwas als ein Dokument ... gekennzeichnet werden soll“, besteht. (Gilt in gleicher Weise natürlich für die Allgemeinen Anhängezahlen der Sprache, des Ortes usw.)

Strenggenommen müssten dann also alle Notationen/ Kennungen für Dokumente/Dokumentarten - genau gleich und genau parallel - zweimal in den Klassifikationen wie der UDC vorkommen: einmal als Kennzeichnung für ein Inhaltsthema, einmal zur Angabe der Form, in der ein Dokument vorliegt. Denn es geht ja um zwei verschiedene Angaben/Kennzeichnungen, die natürlich zwei verschiedenen Angabezwecken dienen. Aber dennoch: ist dies nicht Duplizität/Duplizierung? Könnte man dies lösen, indem man die Dokumentarten (und die anderen Allgemeinen Anhängezahlen) vollständig vorab in den "Allgemeinen Anhängezahlen“ aufführt, mit dem Zusatz (auch hinten bei der jeweiligen Haupttafel): „ohne Klammern können diese Notationen auch als Voll-Notationen für Literatur über die jeweilige Dokumentart verwendet werden“?

Tatsächlich jedoch findet sich derzeit z.B. in der UDC, wie oben ersichtlich, nun de facto eine ganze Reihe von Dokumentarten, von Notationen für spezielle Dokumentarten (etwa zu Film, Foto, Druckwesen) nur „hinten“ im Klassifikationssystem, d.h. nur in der jeweiligen Haupttafel zum Sachgebiet, z. B. bei „77 Photographie“. An diesem Phänomen wäre also wohl auch für eine künftige noch stärkere durchgängige Gleichführung/Parallelführung anzusetzen.

Die in der Studie von Dahlberg erarbeiteten und aufgeführten Dokumentartcodierungen in Gestalt der „Allgemeinen Anhängezahlen der Form“ für die (Charakterisierung der) Dokumentart unterteilen sich mithin in zwei prinzipielle Zielsetzungen:

- Kennzeichnung der materiellen Form des Dokuments, das nachgewiesen ist (Mikrofilm, Fotokopie, Hand- schrift, heute auch: digitales Dokument, z. B. PDF-Datei, ...)

- Kennzeichnung der Darbietungsform der Information in diesem Dokument (Reportage; Polemik; Sachstandsbericht; ... ).

Und diese in der UDC und in verschiedenen anderen Systemen bereits erarbeiteten Dokumentartcharakterisierungen könnte man, selbst auch dann noch, wenn die vorliegende Titelangabe einen ausdrücklichen Hinweis auf die zugrundeliegende Dokumentart enthält, mit der Kennzeichnung durch die in der Dahlberg-Studie vorgeschlagenen Klassifikations- (UDC-) Notationen gut zusammen mit der allgemeinen Inhaltskennzeichnung anzeigen. Der Vorteil einer solchen codierten Dokumentangabe:

- die Klassifikationen dienen ja einer durch Notationen, also unabhängig von den verschiedenen natürlichen Sprachen, angegebenen Dokumentart-Kennzeichnung;

- und einer, die unabhängig ist von der jeweiligen Wortwahl und dem Platz für die Dokumentart-Kennzeichnung in der Titelangabe;

- und sie wollen durch ihre (für denselben Sachverhalt stets gleichen) Notationen das einfache, zusammenführende und aufeinander folgende Ordnen derselben Sachverhalte erreichen, ohne zusätzlichen Sortieraufwand.

Ihre volle Wirksamkeit und Nützlichkeit entfalten daher Dokumentartkennzeichnungen wie die in der DahlbergStudie vorgeschlagenen

- als weiteres Element der „Inhaltserschließung“, für die vertiefte Vorschau auf die Informationsweise der nachgewiesenen Dokumente - also als eine zusätzliche Vorabinformation und Hilfestellung bei der Entscheidung zur Auswahl, ob das angebotene Dokument infrage kommt oder nicht.

- besonders in großen Gesamt-(„Misch“-)Katalogen, in denen viele, unterschiedlichste Arten von Dokumenten gemeinsam verzeichnet sind und daher ein Filter, eine genauere Einengung der Such(ergebniss)e nützlich oder unerlässlich ist.

Dieser Sachverhalt - dass Dokumente nach der Vielzahl ihrer Formen durchaus unter dem Gesichtspunkt als „Dokumentarten“ unterschieden werden können und müssen -, hat wenn auch in unterschiedlicher und manchmal nur partieller Weise seit der Inaugurierung der großen facettierten Klassifikationen zu guter letzt immerhin auch Eingang in die übergreifenden Bemühungen um eine Infrastruktur des Informationsbereichs gefunden, nicht 
überraschender Weise damit auch in die ISO-Normung (so wie er in den früheren weit verbreiteten Vokabularien der Information und Dokumentation schon hätte genannt werden können, aber vielleicht zu selbstverständlich erschien, und erst durch die rasante Entwicklung der Informationstechnik für die Erzeugung digitaler Dokumente die Notwendigkeit formal präziser Definition stärker ins Rampenlicht gerückt ist) - so dass er immerhin jetzt auch definitorisch ansprechbar ist: „document type: class of documents characterized by a specific materiality, purpose or content": so sagt es die im Herbst 2016 erscheinende neue Ausgabe der ISO 5127 „International Standard Foundation and Vocabulary of information and documentation“ (Eintrag 4.4.1.01) ${ }^{12}$, und ähnlich ISO/IEC TR 29166:2011, Document description and processing languages (zuerst 2005).

\section{Welche physische Art von Informationsvermittler/objekt/zugang erwar- tet mich?}

Die gesamte Konzeption der Dokumentartkennzeichnung, der Nutzung der „Allgemeinen Anhängezahlen (der Form)“ als Gegenstand der Dokumentation, der Informationsversorgung, beruht wesentlich darauf, dass - wie eingangs dieses Artikels schon angedeutet - von Anfang an alle Arten von Dokumenten, die gesamte verfügbare Informationsmenge gemeint waren: also als Gegenstand der Dokumentation alle Objekte als „Einheiten (Kombinationen) eines materiellen Datenträgers mit darauf aufgezeichneter Information“ (die ihrerseits in Schrift, Bild, Ton, Hieroglyphen oder wie auch immer kodiert sein kann) - so definieren es, übereinstimmend, alle wichtigen Vokabularien des Informationsbereichs, etwa die DGI-,,Terminologie der Information und Dokumentation“ (1975/2006), die „UNESCO Terminology of documentation“ (1976), die Grundnormen ISO 5127 (2001/2016) oder DIN 31631 Teil 1 (1984), die neue ISO-Thesaurusnorm ISO 25964 Part 1 (2011), der britische Standard BS DD 247 (1998), usw., wie sie früher oder später nach der Erstellung der Studie (1970) von Ingetraut Dahlberg veröffentlicht wurden. Die neuen internationalen Katalogisierungsregeln RDA streben heute ja auch energisch danach, diesen Anspruch ganz konkret einzulösen, indem sie mehr denn je ausdrücklich auf Ver- zeichnung und Beschreibung aller Arten von Dokumenten („Ressourcen“) abheben, nicht mehr nur etwa der klassischen Bibliotheks-Monographien, -Serien und einiger weniger Sondermaterialien. Selbst wenn also die in den Tafeln der der „Allgemeinen Anhängezahlen“ in verschiedenen Klassifikationen aufgeführten Dokumentarten als Codierungen/Kennungen

- vielleicht nicht immer im direkten Zusammenhang mit dem Titel der Ressource zusätzlich angegeben werden müssen,

- so sind sie in jedem Fall u. a. da und dann nützlich, wo in den Angaben der Inhaltserschließung (zu denen die Mehrzahl der Dokumentartangaben aus der DahlbergStudie zweifelsfrei zu rechnen sind) angegeben wird, in welcher Form ein Werk vorliegt oder dass in einem Werk (das vielleicht einen ganz anderslautenden Titel trägt) eine oder mehrere dieser speziellen Dokumentarten behandelt werden.

An einem Punkt allerdings ist die Dahlberg-Studie (S. 197) aber wohl im Irrtum: Der Rat der damaligen UDC-Kollegen, dass eine formale Dokumentartunterscheidung nach einmalig erscheinenden und nach fortlaufend erscheinenden Dokumenten „nicht sehr sinnvoll“ sei, dürfte heute nach wie vor unangemessen erscheinen. Auch wenn diese Unterscheidung natürlich nicht unmittelbar zur Inhaltserschließung beiträgt (man denke aber weiter gehend z.B. an eine lose Flugblattreihe zu einem bestimmten Thema), so ist ihre Bedeutung im gesamten bibliothekarischen Bereich doch ungebrochen, ja hat sich vielleicht sogar noch verstärkt. Relativ klar hervortretend ist auch, wo in der Dahlberg-Studie von 1970 heute Lücken erkennbar sind, wo sie erweitert und ausgebaut werden muss: Das betrifft nicht nur die heute wesentliche Unterscheidung ,analoge/ digitale Dokumente“, sondern auch das gänzliche Fehlen von Dokumentarten, die im Zusammenhang mit Radio/ Fernsehen entstehen (Feature, Nachrichtensendung (als Makrodokument?), ...), aus dem Filmbereich (Drehbuch, Kurzfilm, Schwarz-Weiß-Film, Spielfilm, Hauptfilm, Wochenschau, ... $)^{13}$ und natürlich der digitalen Dokumente, soweit sich dort Formunterschiede ergeben (z. B. nach Dateiformen ?). Die für viele sehr verschiedene Formen (auch ihrer Codierung) vorliegenden DTDs (Document Type Definitions) in der Verarbeitung digitaler Dokumente sind ja heute ein eigens, technisch unverzichtbares Feld der Do-

13 Siehe hierzu z. B. Martha. M. Yee, Moving image materials: genre terms. 1st ed. Washington, D. C.: Library of Congress, Cataloging Distribution Service, 1988. 108 S.
12 International Standard ISO 5127:2016 Information and documen tation: Foundation and vocabulary. Genf: ISO, 2016. 268 S. (Im Erscheinen) [Enthält ca. 2.000 Definitionen im Bereich IuD]. 
kument(art)codierung, mit genauesten Anweisungen für die jeweils spezifische Form.

\section{Dokumentarten in der Internationalen Norm ISO 5127 „Vocabulary of information and documentation“(2016)}

Otlet und die Pioniere der Dokumentation und Informationswissenschaft schufen sich zunächst einen begrenzten Fundus an Grunddefinitionen (speziell die von „Dokument"), die der Grundlegung ihres gesamten Arbeitsprojekts dienten - diese waren jedoch noch keineswegs als Definitionen einzelner Sachverhalte oder Dokumentarten in den Tafeln des System „UDC“ selbst gedacht - und so nur ganz wenige Dokumentarten (,Übersetzung“, .. ) einschlossen, die auch bis dahin bereits in der Bibliotheksund Literaturpraxis gängig waren. ${ }^{14}$

An den Dokumentartkennzeichnungen zeigt sich nun freilich in dieser Hinsicht die deutliche Notwendigkeit, nicht nur Dokumentartbenennungen und -kennzeichnungen bereit zu stellen, sondern diese durch Definition auch zu erklären und in der Verwendung eindeutig zu machen. Eine wesentliche Umsetzung des Konzepts einer breit angelegten, ausgewogenen Auflistung einer größeren Menge von (formalen und inhaltlichen) „Dokumentarten“ enthält hierzu die internationale Norm ISO 5127 „Information and Documentation - Foundation and Vocabulary“, an der die Arbeit 1969, also etwa zeitgleich mit der Dahlberg-Studie, begonnen wurde. Sie wurde in verschiedenen Teilen in den 1970er und 1980er Jahren vorgelegt und 2001 als eine konsolidierte Gesamtausgabe herausgebracht. 2016 wird ihre Folgeausgabe erscheinen (ISO 5127:2016). ${ }^{15}$. Neben den Gebieten „Katalogisierung - Inhaltserschließung/Klassifikation - Institutionen der Information und Dokumentation Konservierung und Restaurierung von Dokumenten“ ist ein Schwerpunkt dieser Norm die komplementäre, terminologische Ergänzung zu den verschiedenen Listen/Klassifikationen von Dokumentarten durch die zugehörige Terminologie, durch Definitionen: aufbauend auf einer sorgfältigen klassifikatorischen Einteilung und begriff-

14 Näheres siehe Ermert, Axel: Begriffsordnung und Terminologie seit jeher unerlässlich für die Dokumentation? In: IWP 59(2008)6-7, S. 369-383, hier S. 372-374.

15 International Standard ISO 5127:2016 Information and documentation: Foundation and vocabulary. Genf: ISO, 2016. 268 S. (Im Erscheinen) [Enthält ca. 2.000 Definitionen im Bereich IuD]. lichen Trennung wird hier auch die Definition für jede einzelne Dokumentart geliefert. Deren Verständnis wird bei den jeweils nur einteilenden Klassifikationen mit ihren Klassenbenennungen ja stets ohne weiteres vorausgesetzt, ist aber keineswegs immer so einheitlich und selbstverständlich anzunehmen wie man oft meint. Diese Definitionen folgen einem einheitlichen Schema, das vor allem auf die genaue Bestimmung der jeweiligen Definition innerhalb des Begriffssystems Wert legt, u. a. mit der durchgängigen Angabe der jeweiligen Ober- und Unterbegriffe in jeder einzelnen Definition. Von Anfang an war in der Norm auch ein eigenes wichtiges Kapitel „Dokumentarten und -teile“ enthalten, das zunächst sich im wesentlichen an den Dokumentarten aus der UDC Klasse 0 (den „klassischen" bibliothekarischen und bibliographischen) orientierte, jedoch auch schon zwei Unterkapitel zu „Künstlerischen bildhaften Dokumenten“ sowie ein Unterkapitel zu „Photographischen Dokumenten“ enthielt. Waren es in der Ausgabe 2001 rund 240 Dokumentarten und Dokumentteile, so wurde dieser Bereich für 2016 erheblich erweitert und tiefergehend und trennschärfer gegliedert, mit nunmehr rund 500 Dokumentarten, 88 Dokumentteilen, und 38 „Makrodokumenten“ (,Akkumulationen von Dokumenten" wie Sammlungen, Nachlässe, .. ), und wird in der bald beginnenden erneuten Überarbeitung der Norm noch ausgebaut werden. Der Schwerpunkt liegt dabei auf Arten konkreter physischer Dokumente - insofern gibt es also viele ,formale‘ Dokumentartbestimmungen nach der materialen Beschaffenheit bzw. dem ,Format' der Dokumentart - , weniger auf Abstraktionsbegriffen, die die Tendenz der inhaltlichen Ausrichtung der Dokumente erfassen (siehe aber unten die Gruppe „Documents by originator and intended purpose“). Das jeweils gewählte grundlegende Definitionskriterium stellt sicher, dass überall, wo das Dokument in seiner Art sowohl analog wie digital beschaffen sein kann, die Definition unabhängig von diesem Merkmal erfolgt, weil das Dokument ja in beiden Formen auftreten kann, und es nur dann heranzieht, wenn das Entscheidende an dem Dokument seine nur analoge oder nur digitale Beschaffenheit ist. (Das ist aber, schaut man genau analytisch hin, überraschend selten der Fall.) Der „Dokumentart“-Abschnitt von ISO 5127:2016 umfasst jetzt folgende Einteilungsmerkmale - und damit einen wichtigen Teil der in den „Allgemeinen Anhängezahlen der Form“ der gegenwärtigen UDC-Ausgabe ${ }^{16}$ ent-

16 UDC Universal Decimal Classification: Standard Edition: Fully updated third edition. 2 Bände (Systematic tables; Alphabetical Index). London: British Standard Edition, 2005. 898, 526 S. 
haltenen Dokumentarten (ungefähre Anzahl der enthaltenen Einträge in Klammern):

- Data media/data carriers (12) (paper, punched card, screen, ... ) (paper, punched card, screen, ... )

- Object documents (42) (prototype, type specimen, monument, ... )(prototype, type specimen, monument, ...)

- Documents by medium (29) (analog document, TV program, hard copy, ... )(analog document, TV program, hard copy, ... )

- Physical production characteristics of documents (15+9) (types of printing and of paper)(types of printing and of paper)

- Document types (25)(25)(25) (unicum/multiplum, published/unpublished, non-booktrade document, monographic/continuing/integrating resource, primary/secondary/tertiary document, types of editions etc.)

- Monographic resources (14) (incunabulum, pamphlet, ... )

- Continuing resources (15) (periodicals, journals, loose-leaf editions, types of newspapers)

- Documents by status (15) (draft, version, reprint, classified document, etc.)

- Derived documents (6) (adaptation, translation, medley, etc.)

- Documents by acquisition status (7) (accession, accrual, estray, etc.)

- Records, archival and legal documents (17) (action copy, archival item, ...)

- Photographic and other optical documents, incl. historic ones (21) (filmstrip, cine film, daguerrotype, ...)

- Artistic graphic documents (28) (line engraving, collotype print, wash drawing, ... )

- Electromagnetic and electronic documents (19) (sound carrier, gramophone record, disk, LP, ... )

- Audiovisual documents by status (13) (director's cut, season, rerun, etc.)

- Patents and other intellectual property documents and their parts (28)

- Documents by originator and intended purpose (16) (government document; artistic document; religious document; trade document; personal document like passport, contract, ...)

- Reference works and data collections $(38+14)$ (dictionaries, thesauri, civil registers, etc.)

- Catalogues and bibliographies (14+9)

- $\quad{ }^{*}$ Intellectual parts of documents (50)

- * Physical parts of documents, book bindings (16 $+11+11)$
- Archival sets of documents (38) (series, personal papers, estates, ...)

${ }^{-}{ }^{*}=$ (Unterabschnitte für die Teile von Dokumenten)

und zwischen diesen das - auch zahlenmäßig - hauptsächliche Unterkapitel

- Documents by main feature (75) (nach Inhalt: minutes, screenplay, diary, ... ), Kommunikationsweg (z. B. Brief), speziellem Format (Telegramm, Tabelle, Notenblatt), etc.).

Die generelle Methodik, in modernen (facettierten) Klassifikationen die Auflistungen jeweils unter dem angemessenen Unterteilungsgesichtspunkt zu führen und diesen auch als „according to“ oder „by“ systematisch anzugeben, wurde - wie eben ersichtlich - in dieser Norm ebenfalls benutzt, ein wichtiger Schritt über bloß aufzählende, alphabetische „Terminologien“ hinaus.

\section{AAT - Art and Architecture Thesaurus}

Und einen weiteren wesentlichen Schritt vorwärts in der Berücksichtigung der präzisen Einteilung und der Bereitstellung der angemessenen Fachausdrücke finden wir in dem als Norm-Vorstufe und systematisches Instrument sehr geeigneten AAT. Im seit den frühen 1980er Jahren aufgebauten AAT „Art and Architecture Thesaurus“"17 finden wir innerhalb der Hauptfacette „Objects“ eine ausführliche Facette „Information and exchange media“ (ca. 1.800 Einträge), die umfangreiches Vokabular für die Benennung (und zur indexierenden Bezeichnung) von Dokumentarten bereitstellt. Zwar sind diese eher für die Schlagwortvergabe für bestimmte Dokumente oder Werke gedacht, soweit sie als Inhalt Gegenstand anderer Werke sind, sie sind aber auch als „Formschlagworte“ geeignet. Unter anderem „Visual works“ ist hier eine wichtige, treffend benannte Gruppe, aber auch besondere Dokumentarten wie „Münzen“ und „Papiergeld“ sind berücksichtigt. Mit diesem Instrument kann also immerhin, wenn schon nicht in der Systematik der speziellen Kennzeichnung als „Formanhängezahl“ eines größeren Klassifikationssystems, so doch in der Verschlagwortung, wenn das gewünscht wird, auf eine große Zahl Einträge eines Indexierungsvokabulars zurückgegriffen werden. Als erster

17 http://www.getty.edu/research/tools/vocabularies/aat/ 
Schritt der Erstellung einer deutschsprachigen Fassung des AAT beim Institut für Museumsforschung (IfM) in Berlin wurde bisher die „Objekt-“Facette (mehr als 17.000 Einträge) des AAT ins Deutsche übertragen; sie enthält reichhaltige Bezeichnungsmengen für Dokumentarten.

Die allgemeine Problematik, die der Beitrag von Ingetraut Dahlberg im Rahmen der umfassenden Neuorientierung, Erweiterung und Präzisierung der dokumentarischen Werkzeuge und der erzeugten dokumentarischen Information (in ihrem Fall: im Rahmen der umfassenden Revision der Universellen Dezimalklassifikation) angesprochen und dafür Lösungen vorgeschlagen hat, ist also auch im heutigen dokumentarischen Geschehen keineswegs bedeutungslos. Ansätze und einzelne Vorstöße $\mathrm{zu}$ seiner Umsetzung liegen vor - die vollständige Einlösung steht noch aus und muss unter den heute stark veränderten technischen Bedingungen der Informationsproduktion, -erschließung und -verbreitung weiter betrie- ben werden. Bei der UDC ist wohl eine Überarbeitung und Erweiterung (und da trifft der Beitrag von Ingetraut Dahlberg passrecht) in der Arbeitsperiode der nächsten Jahre geplant.

Deskriptoren: Formale Erfassung, Klassifikation, Dokumentart, Norm

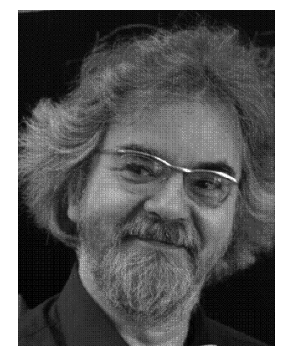

Axel Ermert

DGI-AKTS Arbeitskreis Terminologie und Sprachfragen Institut für Museumsforschung

In der Halde 1

14195 Berlin

a.ermert@smb.spk-berlin.de 